\title{
Modèles botaniques, des modèles scientifiques entre art et science
}

\author{
Botanical models, between art and science
}

\author{
Florence Tessier ${ }^{1}$ \\ ${ }^{1}$ archiviste-paléographe, conservatrice de la bibliothèque de botanique, Muséum national d'Histoire naturelle
}

\begin{abstract}
RÉSUMÉ. La diversité des modèles botaniques en trois dimensions se perçoit d'abord par leurs matériaux, menant à une typologie matérielle. Parmi ceux-ci, il faut compter des modèles en fleurs artificielles jusqu'ici méconnus, dont un exemple est donné par les herbiers artificiels de Marie Fortier. Mais la diversité des modèles botaniques provient aussi de l'évolution de la science botanique elle-même, et de sa relation au monde artistique. Alors que les modèles botaniques anciens deviennent des objets de musée, les modèles botaniques devenus algorithmiques continuent à inspirer les artistes.

ABSTRACT. The diversity of 3D botanical models is perceived at first through their different materials, of which an overview is given. Textile artificial flowers are to be included: some have been crafted as botanical models throughout the $19^{\text {th }}$ century, as reveals the case of Marie Fortier's artificial herbariums. Their diversity also stems from the evolution of the botanical science itself, and its relation to arts. As the old models become museal objects, the algorithmic botanical models still inspire artists.

MOTS-CLÉS. Modèles 3D, modèles botaniques, fleurs artificielles, illustration botanique, Marie Fortier.

KEYWORDS. 3D models, botanical models, artificial flowers, botanical illustration, Marie Fortier.
\end{abstract}

Le lien intime de la botanique avec l'art n'a probablement pas d'égal parmi les autres sciences et a fréquemment été souligné. Le développement à la Renaissance d'une illustration botanique s'attachant à décrire les plantes «ad vivum ${ }^{1}$ ou «d'après nature » a été comme le signe de la naissance de la botanique comme une science moderne basée sur l'observation du vivant. Les herbiers sont un outil essentiel du botaniste. L'utilisation simultanée des termes «herbarium » et « herbier » pour les collections d'images de plantes comme pour les collections de plantes sèches souligne que les unes et les autres sont concurremment pensées et utilisées comme substituts du sujet naturel et vivant, et en tant que tels outils et supports principaux de la botanique aux XVII ${ }^{\mathrm{e}}$ et XVIII ${ }^{\mathrm{e}}$ siècles.

L'illustration botanique avec ses codes spécifiques fixés au début du XIX ${ }^{\mathrm{e}}$ siècle ${ }^{2}$, qui reflètent son lien étroit aux herbiers, a gagné une place éminente dans le monde visuel moderne et continue de susciter l'intérêt du public comme des artistes. Elle reste de nos jours une inspiration forte dans les arts visuels et décoratifs.

Les représentations en trois dimensions, qui ont pourtant partagé pendant une large part de l'histoire de la botanique européenne la fonction scientifique et didactique des illustrations, sont loin d'avoir acquis la même reconnaissance. Malgré trois siècles d'ancienneté, la variété des techniques employées, et leur large utilisation dans l'enseignement et la médiation de nombreuses branches de la biologie au $\mathrm{XIX}^{\mathrm{e}}$ et au $\mathrm{XX}^{\mathrm{e}}$ siècle, les modèles botaniques en trois dimensions restent peu connus, même s'ils commencent à susciter un nouvel intérêt en tant que représentations visuelles d'une

\footnotetext{
${ }^{1}$ Thomas Balfe, Joanna Woodall et Klaus Zittel, Ad vivum? : Visual Materials and the Vocabulary of Life-Likeness in Europe before 1800, s.l., Brill, 2019.

${ }^{2}$ Pierre-Yves Lacour, " De la divergence art/science. L'image naturaliste entre 1720 et 1820 », Études sur le XVIIle siècle, 2015, vol. 42, p. 193- 207.
} 
pensée scientifique et d'un discours pédagogique. De nombreuses collections de modèles sont encore en cours de redécouverte et d'inventaire dans les institutions variées qui en conservent; et l'existence même de certains types de modèles, tels les herbiers de plantes artificielles en textile, reste complètement ignorée.

Un rappel des différents types matériels de modèles botaniques, abordés sous l'angle du matériau utilisé, sera l'occasion d'un regard plus poussé sur les modèles en fleurs artificielles. Nous verrons ensuite comment les modèles, d'abord issus du monde artistique par leur mode de fabrication et leur rôle iconique, s'en dégagent en s'adaptant à l'évolution de la science botanique, pour y revenir lorsque leur signification scientifique s'atténue.

\section{Modèles botaniques en trois dimensions : une typologie matérielle}

«Les dessins coloriés sont utiles; l'exécution des plantes en relief aura une utilité d'un autre genre et sera surtout recherchée pour les plantes grasses, telles que les ficoïdes, cierges, euphorbes, joubarbes, champignons, etc, dont on ne peut conserver les formes et les caractères dans les herbiers, et que l'on ne parvient même jamais à bien dessécher. Il seroit surtout avantageux de fixer par une imitation exacte la forme et la structure des champignons, dont la plupart n'ont qu'une existence éphémère, et qui, dès lors, ne peuvent être présentés avec ordre aux élèves dans les démonstrations botaniques. »

Contrairement à ce que suggère ici Antoine-Laurent de Jussieu en $1791^{3}$, les représentations de plantes en trois dimensions ne sont pas restées cantonnées à ces cas particuliers où la mise en herbier trouve ses limites, mais, à l'instar des arts graphiques, ont servi à illustrer tous les types de plantes.

La diversité des modèles botaniques s'exprime également dans les matériaux utilisés, dans leur mode de production, dans les intentions de leurs auteurs et dans les usages qui ont pu en être faits.

La terre-cuite semble avoir été le premier matériau utilisé : les plus anciens modèles botaniques seraient les fruits en terre-cuite faisant partie des collections envoyées d'Indonésie au grand-duc de Toscane Cosme III de Médicis par le botaniste Georg Eberhard Rumphius (1624-1702).

Né en Italie dans un contexte religieux (ornements d'autels, ex-votos de guérisons) l'art de modeler et mouler la cire se diversifie au XVII ${ }^{\mathrm{e}}$ siècle vers la production de modèles anatomiques mais aussi botaniques. La constitution d'ateliers renommés et de larges collections encore conservées à Bologne, Florence ou Pise contribuent au XVIII ${ }^{\mathrm{e}}$ siècle à sa diffusion dans les pays proches. Des artistes français illustrent cet art de la céroplastie au tournant du XVIII ${ }^{\mathrm{e}}$ et du XIX ${ }^{\mathrm{e}}$ siècles : le Muséum national d'Histoire naturelle conserve ainsi les œuvres d'André Pierre Pinson (1746-1828) avec une collection de cinq cents champignons produite vers 1794-1800 dans l'atelier de céroplastie de l'Ecole de Santé de Paris ${ }^{5}$; et celles de Louis Marc Antoine Robillard d'Argentelle

\footnotetext{
${ }^{3}$ Charles de Villette, « Projet d'établir en France une manufacture de végétaux artificiels, [...] ; d'après les nouveaux procédés de T.-J. Wenzel, fleuriste de la Reine, rédigé par L. F. Jauffret, In-8vo de 136 p. A Paris, 1790 ... ", L'esprit des journaux, francais et etrangers, avr. 1791p. 152- 159p.

${ }^{4}$ Mauro Raffaelli (ed.), II Museo di storia naturale dell'Università degli studi di Firenze. II, Le collezioni botaniche = The Museum of natural history of the University of Florence. II, The botanical collections, Firenze, Italie, Firenze University Press, 2009, p. 7-9.

${ }^{5}$ Michel Lemire, Anne-Marie Slezec et Georges Boulinier, « Un anatomiste sculpteur sur cire à l'époque révolutionnaire : André Pierre Pinson (1746-1828) » dans Scientifiques et sociétés pendant la Révolution et l'Empire (114e Congrès National des Sociétés Savantes, Section Histoire des Sciences et des Techniques, Paris, 1989), Comité des travaux historiques et Scientifiques., Paris, 1990, p. 325- 337.
} 
(1777-1828), la collection de fruits exotiques modelée à l'Île Maurice entre 1802 et 1826 puis exposée à Paris sous le nom de «Carporama » ${ }^{6}$ avant de rejoindre les collections du Muséum en 1867. Une autre collection de champignons en cire conservée au Muséum a été diffusée en plusieurs exemplaires par le mycologue autrichien Leopold Trattinick (1764-1849).

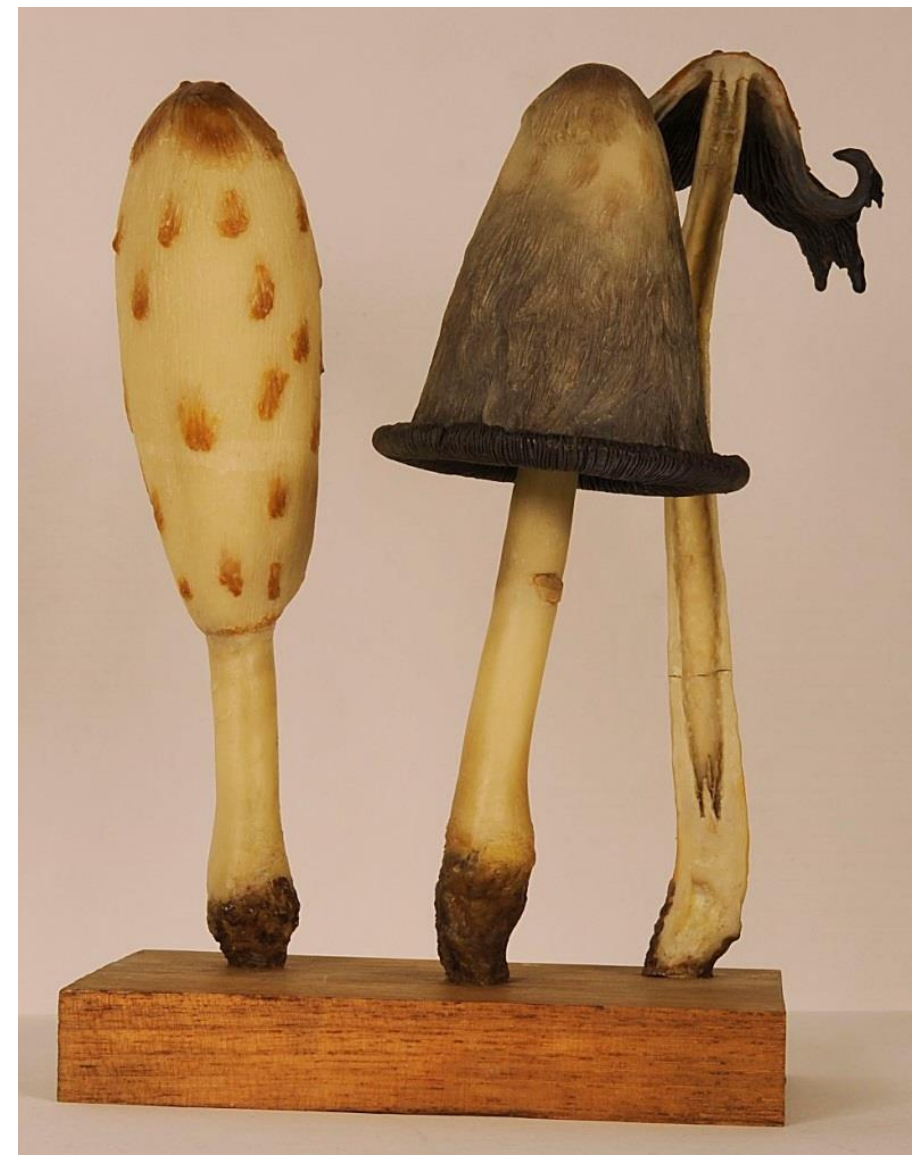

André Pierre Pinson, Agaricus typhoides (Bullier) , 1802-1817, cire colorée modelée, Muséum national d'histoire naturelle, OA 1500 (186) ; crédit : Muséum national d'histoire naturelle

Matériau plus modeste, le plâtre est également employé en lien avec la production de modèles de cire, comme en témoigne la collection de modèles de champignons réunie entre 1850 et 1890 par Jean Baptiste Barla à Nice, qui comprend à la fois les moules en plâtre et leurs tirages en cire, plâtre ou papier-mâché, mis en couleurs par l'aquarelliste Vincent Fossat ${ }^{7}$. En Italie, France, Allemagne et ailleurs, de nombreuses collections pomologiques ont également été moulées en cire pour illustrer les variétés de fruits dans le cadre d'un enseignement agronomique.

\footnotetext{
${ }^{6}$ Monique Keraudren-Aymonin et Gérard G. Aymonin, « Une œuvre scientifique et artistique unique: le Carporama de L. M. A. de Robillard d'Argentelle », Bulletin de la Société Botanique de France. Lettres Botaniques, 1 janvier 1984, vol. 131, $\mathrm{n}^{\circ}$ 4- 5, $\mathrm{p}$. 243- 246.

${ }^{7}$ Brigitte Chamagne-Rollier, "La collection de moulages de champignons de Jean-Baptiste Barla », La Lettre de l'OCIM. Musées, Patrimoine et Culture scientifiques et techniques, 25 juin 2014, n 153.
} 


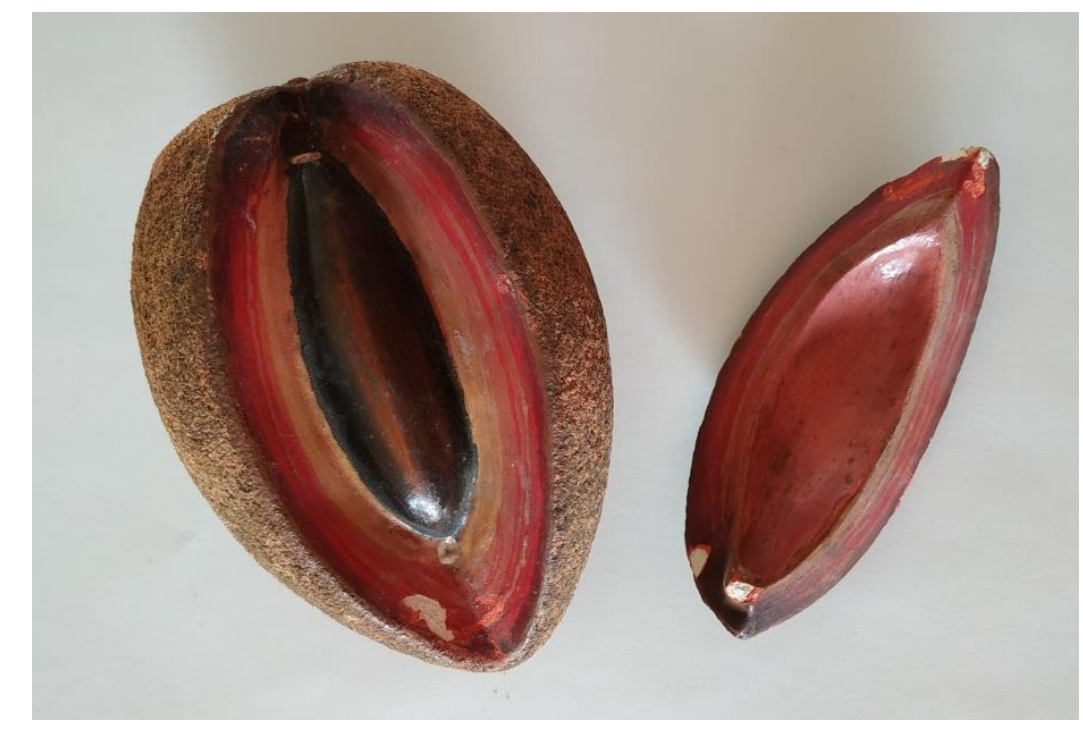

Pouteria sapota ou Sapote, non signé, non daté, plâtre peint, Muséum national d'Histoire naturelle ; crédit : F. Tessier.

Euvres uniques également, les modèles zoologiques et botaniques des verriers allemands Léopold Blashka (1822-1895) et son fils Rudolf Blashka (1857-1939). Les plus connus sont les quatre mille modèles en verre reproduisant à taille réelle des plantes, leurs fleurs et autres détails d'anatomie végétale réalisés de 1887 à 1936 sur une commande de l'Université de Harvard ${ }^{8}$; mais d'autres modèles botaniques en verre sont conservés dans divers musées allemands.

On peut aussi noter le cas étonnant du Dr Dillon Weston ( 1899-1953) qui s'adonne en amateur au travail du verre pour la réalisation d'une collection de modèles de champignons microscopiques et de plantes attaquées par ces pathogènes, aujourd'hui conservée au Whipple Museum of the History of Science de l'Université de Cambridge?

Le papier-mâché (ou plus exactement une pâte composite dont la pâte à papier n'est qu'un élément) a été adopté pour une production en série grâce à son faible coût et la facilité de sa mise en œuvre: le Dr Louis Auzoux est le pionnier et l'exemple français de cette production industrielle avec sa fabrique qui resta en activité de 1828 à $1990^{10}$. Sa première activité était la production de modèles d'anatomie humaine et animale, mais il y rajoute des modèles botaniques à partir des années 1860. Ce mode de fabrication est copié par des firmes allemandes dont la plus connue est celle fondée par Robert Brendel à Breslau en 1866, et dont la diffusion en France et dans le monde est aussi large que celle des modèles Auzoux ${ }^{11}$.

Egalement diffusés par des éditeurs de matériel scolaire comme Deyrolles, les modèles Brendel et Auzoux sont en effet considérés comme faisant partie du matériel de base pour l'enseignement des

\footnotetext{
${ }^{8}$ Richard Evans Schultes, William A. Davis et Hillel S. Burger, The glass flowers at Harvard, Cambridge, Etats-Unis d'Amérique, Botanical Museum of Harvard University, 1992, vii+118 p.

${ }^{9}$ Henry T. Tribe, « The Dillon-Weston glass models of microfungi », The Mycologist, 1998, vol. 31, p. $169-173$.

${ }^{10}$ Nicolas Chanal, L'anatomie clastique du Docteur Auzoux, une entreprise au XIXème siècle, Thèse d'exercice, École nationale vétérinaire d'Alfort, France, 2014, 140 p.

${ }^{11}$ Graziana Fiorini, Luana Maekawa et Peter Stiberc, "La "Collezione Brendel” di Modelli di Fiori ed Altri Organi Vegetali del Dipartimento di Biologia Vegetale dell'Università degli Studi di Firenze », Museologia scientifica, 1 janvier 2005, vol. 22, p. 249- 273.
} 
sciences au niveau primaire et secondaire ${ }^{12}$, et se retrouvent aujourd'hui dans de nombreuses collections de matériel pédagogique ancien.

Ces divers types de modèles botaniques ont fait l'objet de recensements souvent basés sur des collections muséales $^{13}$. Il en existe un de plus, les modèles de plantes en tissu, dont la quasidisparition de nos jours explique qu'ils soient passés inaperçus.

\section{Fleurs de cire, fleurs de tissu : des salons aux musées}

Ann B. Shteir ${ }^{14}$ a décrit comment le modelage de fleurs en cire a pu devenir dans l'Angleterre victorienne à la fois un art de salon et un puissant outil de médiation scientifique. Suivant un exemple donné par les cours royales dès le XVIII ${ }^{\mathrm{e}}$ siècle, le goût pour les fleurs de cire pénètre fortement la décoration intérieure et la mode, et leur fabrication est promue auprès des jeunes filles et femmes de l'élite comme une activité artistique qui se double d'une leçon de botanique. L'industrie des fleurs artificielles en cire créée par cette mode se soutient au-delà du déclin de cet engouement grâce aux dioramas des musées d'histoire naturelle. Le National Museum Wales a ainsi continué à commanditer des modèles de cire jusqu'au $\mathrm{XX}^{\mathrm{e}}$ siècle.

Un rôle similaire est tenu en France par la fabrication de fleurs artificielles en tissu. Né en Italie et introduit en France au XVII ${ }^{\mathrm{e}}$ siècle $^{15}$, cet artisanat d'abord pratiqué dans les couvents devient une industrie et un commerce fournissant les plus riches pour la décoration intérieure religieuse et mondaine, les fêtes et spectacles, et la mode. C'est aussi un passe-temps pratiqué par quelques femmes de l'aristocratie.

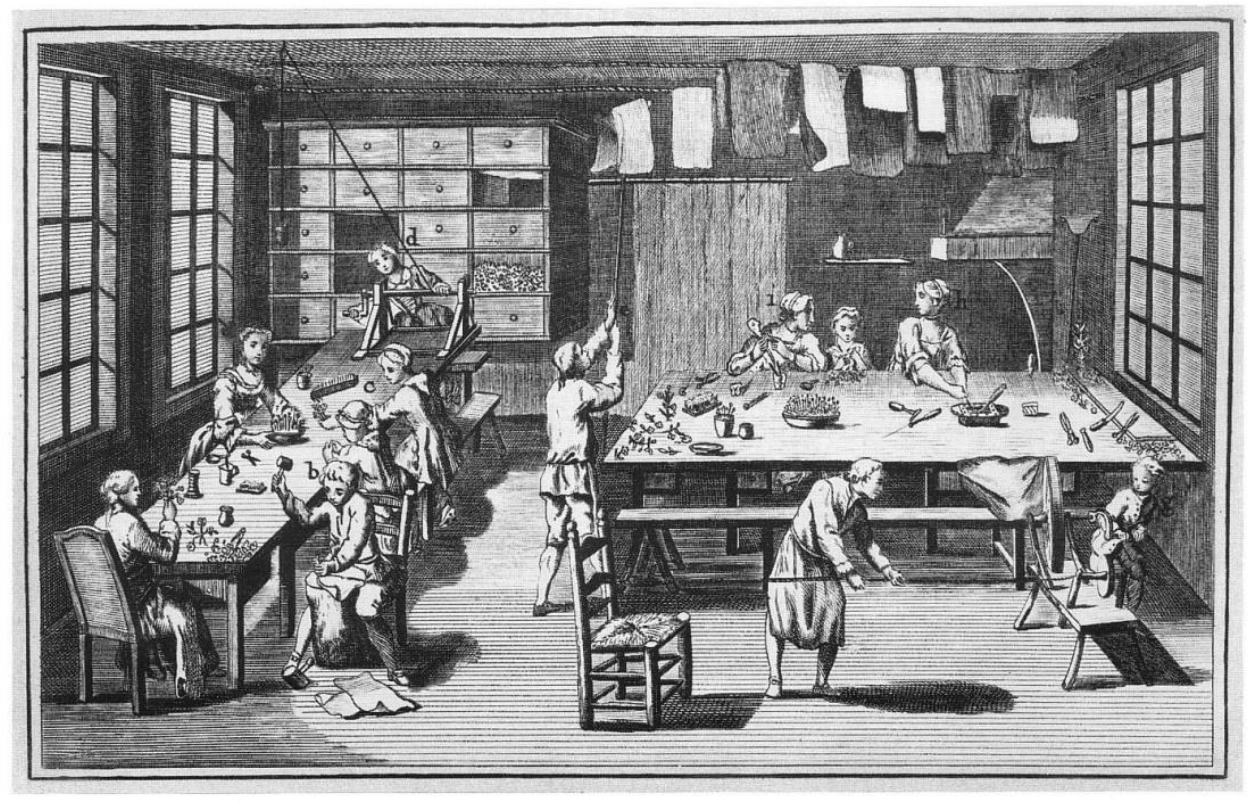

Encyclopédie, ou Dictionnaire raisonné des sciences, des arts et des métiers. Planches. t. 4, Fleuriste artificiel, pl. 1 : atelier, 1751 ; crédit : Gallica.bnf.fr / Bibliothèque nationale de France.

\footnotetext{
${ }^{12}$ William Tuckwell, « Science-Teaching in Schools », Nature, 1869, vol. 1, p. 18- 20.

${ }^{13}$ Anne-Marie Bogaert-Damin, Voyage au cœur des fleurs: modèles botaniques et flores d'Europe au XIXème siècle, Namur, Belgique, Presses universitaires de Namur, 2007, 239 p.

${ }^{14}$ Ann B. Shteir, " "Fac-similes of nature : Victorian Wax flower modelling ", Victorian Literature and Culture, septembre 2007, vol. $35, n^{\circ} 2$, p. 649- 661 .

${ }^{15}$ Denis Diderot, « Fleuriste artificiel » dans Encyclopédie, ou dictionnaire raisonné des sciences, des arts et des métiers ..., Paris, France, Briasson, 1751, vol.6, p. 866.
} 
Cette industrie grandit à Paris au cours du $\mathrm{XIX}^{\mathrm{e}}$ siècle jusqu'à employer des dizaines de milliers d'ouvrières et ouvriers dans les différentes étapes de sa production. Elle reste parallèlement considérée comme un loisir respectable et utile pour les femmes des classes supérieures, et suscite une littérature de manuels pratiques à leur intention ${ }^{16}$.

Son lien avec la science botanique est cependant resté quasi ignoré ${ }^{17}$. Le medium des fleurs artificielles a pourtant été proposé dès la fin du XVIII ${ }^{\mathrm{e}}$ siècle comme support d'enseignement pour la botanique.

Mme de Genlis, précepteur des enfants de Philippe d'Orléans propose dans un «Discours de la botanique considérée relativement à l'éducation » de faire faire aux jeunes filles des herbiers de fleurs artificielles ${ }^{18}$; elle dit en avoir fait un elle-même de sept cent plantes exotiques, formant ainsi un « cabinet de botanique très curieux » dans les années 1780. Cette collection, présentée à Buffon, lui aurait donné l'envie de s'en inspirer pour le Cabinet royal d'histoire naturelle ${ }^{19}$.

« L'auteur de cet ouvrage a fait, il y a vingt-trois ou vingt-quatre ans, près de mille plantes artificielles, presque toutes exotiques, qui formoient un cabinet qui a été vu par un grand nombre de personnes ; ces plantes, étoient d'une imitation que M. de Buffon trouva si parfaite, qu'il parut regretter vivement de n'avoir pas de fonds pour faire cette entreprise en grand, au Cabinet d'histoire naturelle ; il auroit voulu faire une galerie remplie de ces fleurs, pour servir à l'étude de la botanique durant l'hiver. Pour conserver toujours ces plantes placées dans des caisses, il faut les couvrir de châssis de gaze ou de canevas, le tout recouvert d'enveloppes détachées de toile cirée, et, quand on les découvre, ne pas les exposer au soleil. »

Quelques années plus tard, en 1790, l'idée de constituer une exposition de plantes artificielles est proposée par Thomas Joseph Wenzel, fabricant de fleurs artificielles et ancien fournisseur de MarieAntoinette $^{20}$. Les débuts de la Révolution et la promotion d'un train de vie plus sobre et vertueux sont sans doute préjudiciables à son industrie de luxe, qui a employé jusqu'à deux cents ouvriers et ouvrières ; il tente ainsi de mettre son art au service de la science et de l'éducation du peuple. Bien que soutenu par des journalistes et, si l'on en croit leurs articles ${ }^{21}$, par d'éminentes figures scientifiques, le projet n'entre pas dans les priorités du gouvernement révolutionnaire.

\footnotetext{
${ }^{16}$ Elisabeth Celnart, Manuel du fleuriste artificiel ou l'art d'imiter d'après nature toute espèce de fleurs, Paris, Librairie encyclopédique de Roret, 1829.

${ }^{17}$ Florence Tessier, « Les herbiers artificiels de Marie Fortier », s.l.

${ }^{18}$ Stéphanie-Félicité Du Crest Genlis, Discours moraux sur divers sujets, et particulièrement sur l'éducation ; par Madame de Genlis. Troisième édition, revue, corrigée, et augmentée d'un nouveau discours intitulé: projet d'une école rurale, France, 1802.

${ }^{19}$ Stéphanie-Félicité Du Crest Genlis, Maison rustique, pour servir à l'éducation de la jeunesse, ou, Retour en France d'une famille émigrée: ouvrage où l'on trouve toutes les instructions nécessaires pour bâtir une maison de campagne ..., Paris, France, Chez Maradan, 1810.

${ }^{20}$ Louis-François Jauffret, Projet d'établir en France une manufacture de végétaux artificiels...rédigé par Louis-François Jauffret,...d'après les nouveaux procédés de T.-J. Wenzel, fleuriste de la reine, Paris, France, 1790, 120 p.

${ }^{21}$ C. de Villette, « Projet d'établir en France une manufacture de végétaux artificiels, [...] ; d'après les nouveaux procédés de T.-J. Wenzel, fleuriste de la Reine, rédigé par L. F. Jauffret, In-8vo de 136 p. A Paris, 1790 ... », art cit.
} 


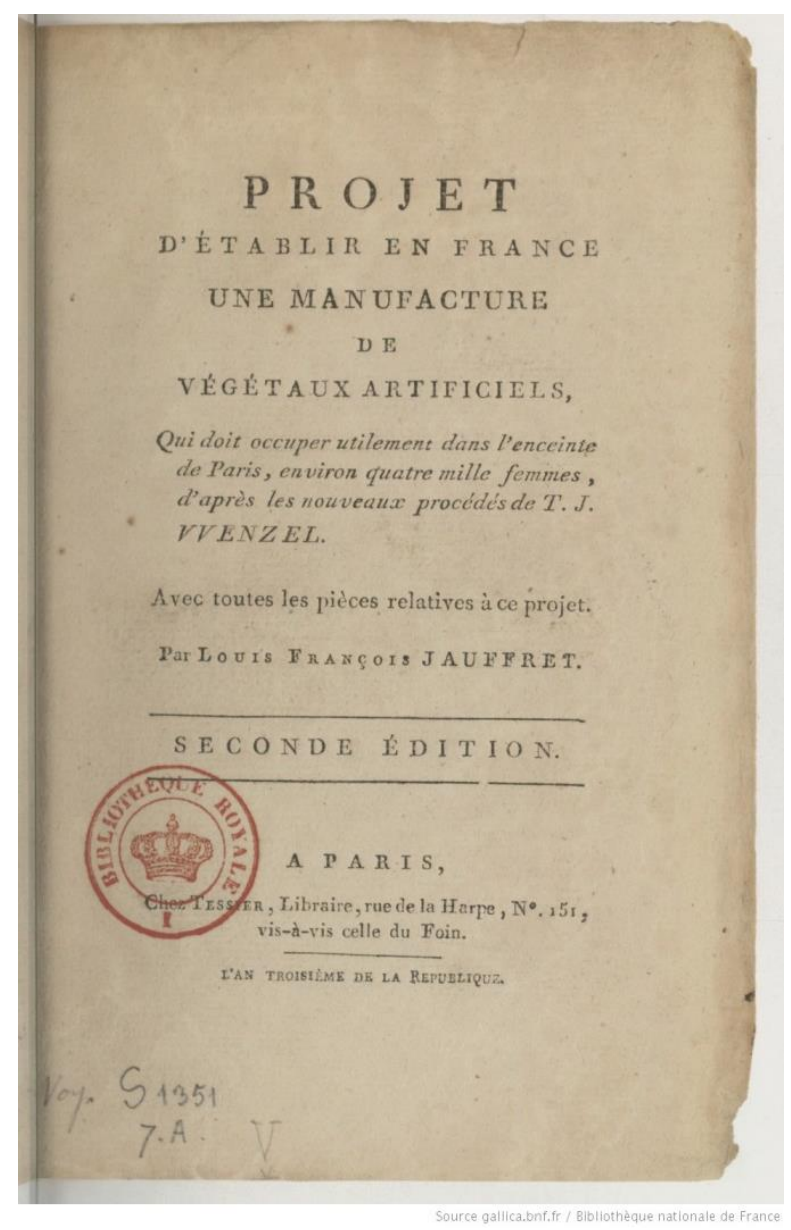

Jauffret, Louis-François (1770-1840). Projet d'établir en France une manufacture de végétaux artificiels... Paris, 1794 ; crédit : Gallica.bnf.fr / Bibliothèque nationale de France.

L'idée de produire des fleurs artificielles pour la botanique accompagne discrètement le développement de l'industrie des fleurs artificielles au cours du XIX ${ }^{\mathrm{e}}$ siècle, et on peut la suivre dans les rapports sur les expositions annuelles des produits de l'industrie : à partir de 1839 une catégorie du concours de l'Exposition est réservée aux «fleurs pour la botanique », séparément des fleurs artificielles pour la mode. Bien que les lauréats de ces expositions soient souvent décrits comme ayant été formés ou encouragés par d'éminents botanistes ${ }^{22}$, on ne trouve pourtant pas mention de ce type de modèles dans la littérature scientifique contemporaine, mis à part une présentation de plantes artificielles à la Société de pharmacie par Mme Vény, un de ces lauréates. Le rapport, très favorable, estime que « madame Vény a fait pour l'étude du règne végétal ce que le Dr Auzoux a fait $[. .$.$] pour la science de l'anatomie { }^{23}$. Les auteurs d'ouvrages d'enseignement de la botanique ne mentionnent que rarement les modèles en trois dimensions; et jamais les fleurs artificielles.

C'est à la fin du XIX ${ }^{\mathrm{e}}$ siècle qu'une fleuriste ${ }^{24}$, Marie Fortier, devient « fabricante d'herbiers artificiels ${ }^{25}$ destinés à l'enseignement de la botanique, qui rencontrent un certain succès. Ces

\footnotetext{
${ }^{22}$ Exposition nationale. 1849. Paris, « Fleurs de botanique artificielles » dans Rapport du jury central sur les produits de l'agriculture et de l'industrie exposés en 1849, Paris, Imprimerie nationale, 1850, vol.3, p. 647.

${ }^{23}$ Guibourt, Chattin et Cap, « Rapport fait le 2 décembre 1846 sur des plantes artificielles présentées par Mme Vény », Journal de pharmacie et de chimie, 1847, vol. 11, p. 39.

${ }^{24}$.Le mot de fleuriste au XIXe siècle désigne en priorité les ouvrières et commerçants en fleurs artificielles, plutôt que les marchandes et marchands de fleurs naturelles et de fleurs coupées.
} 
herbiers sont des fleurs artificielles de plantes sauvages à la fois communes et utiles, présentés montées sur des feuilles et étiquetées comme des herbiers.

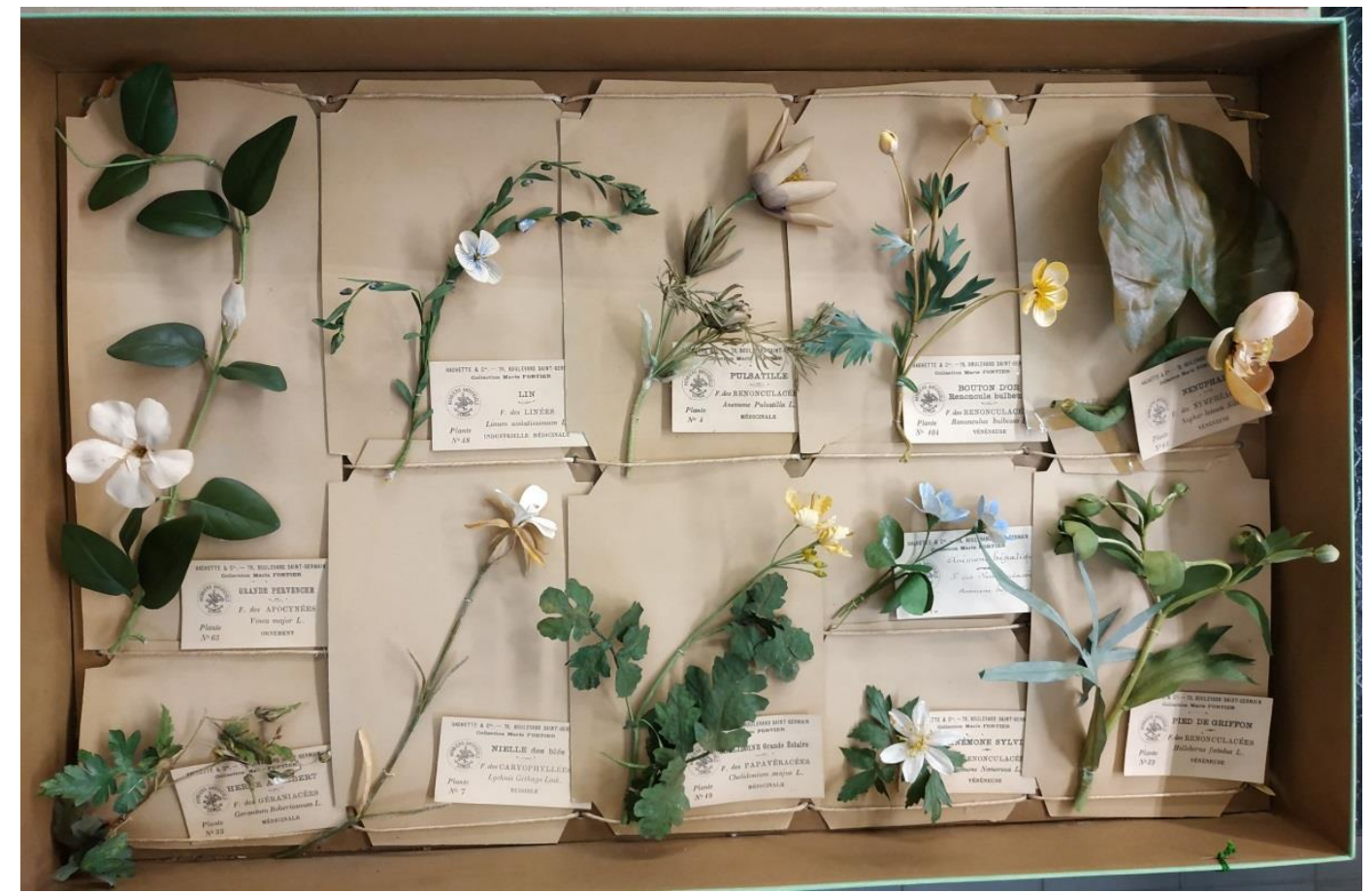

Marie Fortier et Hachette, Herbiers artificiels. 1887-1889. Textile, matériaux divers. Instituto Cardenal Cisneros, Madrid ; crédit : F. Tessier.

Elle commence à les faire connaître vers 1883 en les présentant dans des expositions organisées par des sociétés d'horticulture locales ou par la Société nationale d'Horticulture, obtenant de nombreux prix. Elle publie peu après une brochure présentant son travail ${ }^{26}$. En 1887 elle se tourne vers le réseau des botanistes: elle s'inscrit aux cours du Laboratoire de botanique pratique du Muséum où elle est citée comme une « élève assidue », et elle est reçue quelques mois plus tard à la Société botanique de France, une institution qui a de forts liens avec les botanistes du Muséum, et qui n'a jusqu'alors accepté que très peu de femmes.

Le public visé par Marie Fortier n'est pourtant pas le monde scientifique lui-même : elle destine en effet ses herbiers artificiels à l'enseignement primaire et rural . Le matériel pédagogique est un marché porteur dans cette période de développement de l'enseignement public, où les sciences prennent une place accrue au programme de l'enseignement primaire avec une pédagogie fortement basée sur les leçons de choses et autres musées scolaires. Pour y trouver sa place, Marie Fortier conclut en 1887 un contrat avec Hachette, espérant leur confier la promotion et la commercialisation de ses herbiers, et se consacrer à la fabrication. Elle est rapidement insatisfaite du résultat, estimant qu'elle trouve d'elle-même davantage de clients potentiels que ne lui en apporte Hachette. Avant même ce contrat, elle avait en effet reçu des commandes de la Ville de Paris pour ses écoles primaires : celles-ci se poursuivront quasi annuellement de 1886 à 1908.

Elle a également vendu des fleurs artificielles pour décorer les dioramas du Musée forestier de la Ville de Paris créé en 1891 et installé sur l'île de Bercy du lac Daumesnil au bois de Vincennes.

\footnotetext{
${ }^{25}$ Société botanique de France, "Séance du 10 février 1888 », Bulletin de la Société botanique de France, 35 (1888), $\mathrm{n}^{\circ} 2, \mathrm{p}$. 103- 105.
}

${ }^{26}$ Marie Fortier, Botanique, Notice sur les collections artificielles, s.I., Impr. de Chaix, 1885. 


\section{Epistémologie des modèles botaniques}

Des fruits en cire de Rumphius (1624-1702) aux algorithmes actuels, les modèles jouent un rôle central et croissant dans l'histoire des sciences, suscitant une abondante réflexion sur leur définition, leur nature, leurs fonctions sémiologiques et épistémologiques et leur évolution historique depuis les modèles matériels ou physiques vers des modèles de plus en plus formels ${ }^{27}$.

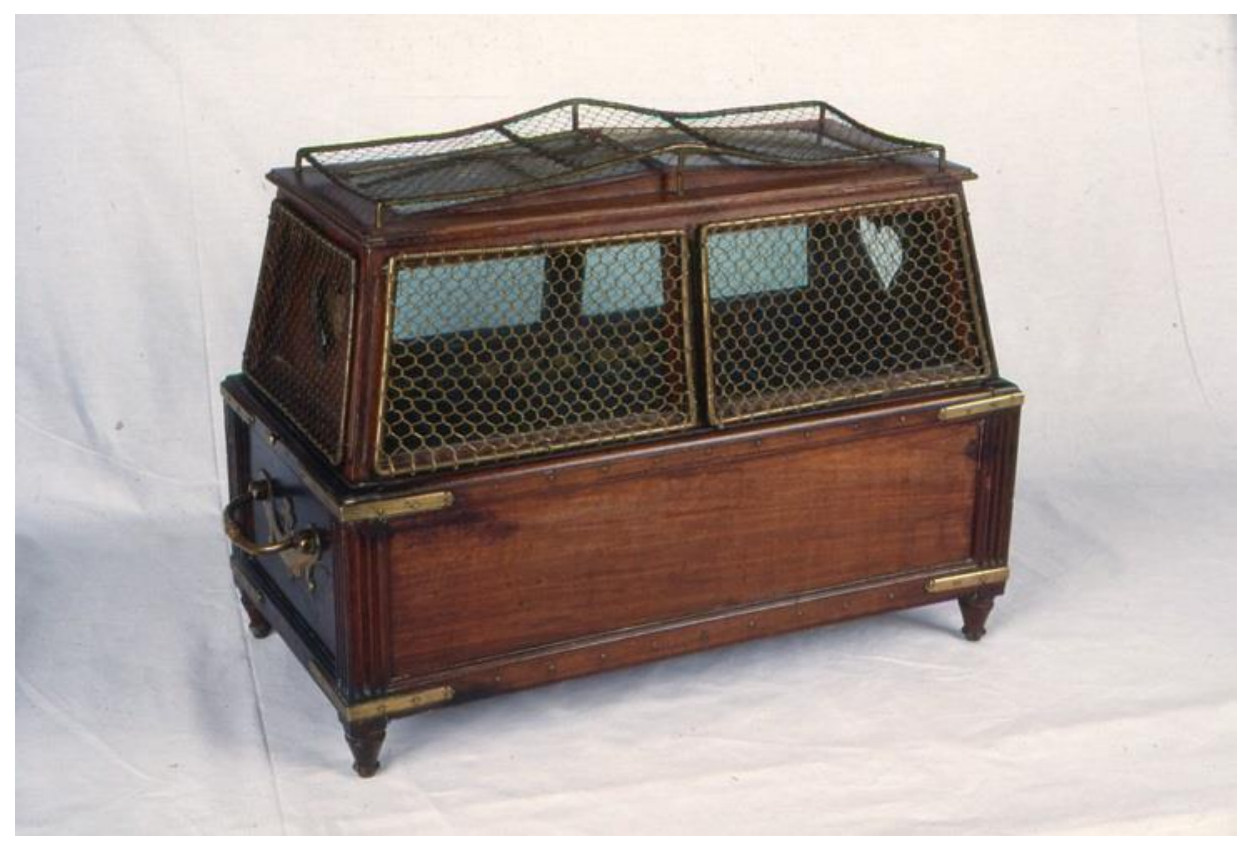

André Thouin (conception), I. Ledoux (réalisation), Maquette de serre portative, 1781, Muséum national d'histoire naturelle, OA 321 ; crédit : Muséum national d'histoire naturelle

$\mathrm{Au}$ regard des typologies proposées par des épistémologues, les modèles botaniques que nous évoquons ici font partie des modèles physiques ${ }^{28}$; ce sont historiquement les premiers modèles utilisés en sciences et dont l'exemple le plus souvent donné est la maquette ou modèle réduit. ${ }^{29}$ Représentation simplifiée d'un objet représenté (son référent pour le sémiologue), le modèle botanique est de la même façon un objet tangible. Il est conçu de façon à reproduire les propriétés visuelles de son sujet : formes, couleurs, détails anatomiques externes et souvent internes, y ajoutant parfois certains aspects tactiles (pubescence).

Les fonctions des modèles ont servi de base à Frank Varenne ${ }^{30}$ pour établir une large typologie des modèles : pour nos modèles botaniques, elles sont essentiellement de faciliter l'observation, en s'extrayant des contraintes du vivant, sa temporalité et sa caducité ; et de faciliter la compréhension et la mémorisation de ce qui est observé.

\footnotetext{
${ }^{27}$ Roman Frigg et Stephan Hartmann, « Models in Science.pdf » dans The Stanford Encyclopedia of Philosophy, s.I., 2006, p.

${ }^{28}$ Franck Varenne, « Epistémologie des modèles et des simulations » dans Les modèles, possibilités et limites, Paris, Editions Matériologiques, 2014, p. 13- 46.

29 . Le Muséum national d'Histoire naturelle possède aussi des modèles réduits de ce type, notamment la collection de maquettes d'instruments agricoles et horticoles d'André Thouin.

${ }^{30}$ Franck Varenne, "Histoire de la modélisation : quelques jalons » dans Modélisation, Succès et Limites : actes du colloque organisé par le CNRS et l'Académie des technologies, 6 décembre 2016, Pdf diffusé en ligne., s.l., 2018, p. 9- 35.
} 


\section{De l'imitation à l'illustration de la nature}

«Depuis que la coquetterie des modes a tant multiplié ces fleurs artificielles qui sont entrées dans les parures des femmes, l'art du fleuriste s'est appliqué à travailler aussi pour l'honneur et les progrès de la botanique [...] Jamais l'art n'imita mieux la nature. Ce n'est ni le mensonge grossier de la gravure ni l'aspect mort de l'herbier. Ici tout est vivant; la plante semble végéter ; et d'un coup d'œil vous saisissez son ensemble et ses détails. » ${ }^{31}$

Dans une étude sur les relations entre modèles et théorie scientifique dans la biologie du XIX siècle, David Ludwig propose une autre approche de la définition du modèle ${ }^{32}$. Il estime en effet que seul son usage fait d'un objet un modèle : «Les modèles matériels doivent être compris dans le contexte de la pratique scientifique : un objet est considéré comme un modèle si, et seulement si, il est utilisé de façon spécifique ${ }^{33}$. Incluant par ailleurs les illustrations parmi les modèles, il soutient qu' " aucun caractère physique ou fonctionnel général ne distingue des statues ou des dessins qui sont des modèles [...], de statues ou de dessins qui ne sont pas considérés comme des modèles ».

Cette définition suggère que des images ou des objets peuvent, selon leur usage, être alternativement considérés comme des œuvres d'art ou des modèles.

De fait, nous constatons que certains modèles de botanique ont été créés par des artistes ou des artisans d'art, qui produisaient également des œuvres non destinées à servir de modèles. Ainsi le céroplaste André-Pierre Pinson était-il également auteur de portraits en cire $^{34}$; les fabricantes de fleurs artificielles primées dans les expositions des produits de l'industrie concourent aussi bien dans la catégorie «fleurs artificielles pour la botanique » que dans celle des fleurs pour la mode et la décoration.

Les modèles botaniques, en tant que représentations visuelles, partagent avec les œuvres d'art qui leur sont contemporaines une démarche d'imitation de leur référent ; ils sont donc évalués selon des critères communs avec celles-ci : perfection de l'imitation, compétences techniques exigées par leur réalisation, et bien sûr aspect esthétique.

Il nous semble pourtant que les modèles botaniques ont connu une évolution dans leur rapport avec l'art, et que celle-ci est étroitement liée à l'évolution de la science botanique elle-même.

Anne-Marie Bogaert-Damin ${ }^{35}$ souligne justement que «rares sont les modèles botaniques [...] qui ont été conçus dans un but purement esthétique comme des œuvres d'art», soulignant l'importance du but recherché dans la conception du modèle.

En effet la conception du modèle suppose un choix entre les aspects du réel qu'il représente : « Il ne représente pas l'ensemble des propriétés du réel, mais seulement certaines des propriétés » ${ }^{36}$. Ces choix dépendent de l'intention de son auteur, du discours scientifique qui est signifié .

\footnotetext{
${ }^{31}$ François Levaillant, Second voyage dans l'intérieur de l'Afrique par le cap de Bonne-Espérance, dans les années 1783,84 et 85. Tome 2, Paris, H. J. Jansen, 1794, p. 162.

32 David Ludwig, "Mediating Objects: Scientific and Public Functions of Models in Nineteenth-Century Biology », History and Philosophy of the Life Sciences, 2013, vol. 35, n², p. 139- 166.

33 Ibid

${ }^{34}$ Alain Corbin, Jean-Jacques Courtine et Georges Vigarello, Histoire du corps: De la Renaissance aux Lumières, s.l., Seuil, 2005, $668 \mathrm{p}$.

${ }^{35}$ A.-M. Bogaert-Damin, Voyage au cour des fleurs, op. cit.
} 
Tandis que le changement de matériau crée le modèle : « un corps n'est pas fait en cire, une fleur pas en papier mâché, une anémone de mer pas en verre ${ }^{37}$, toutes les autres propriétés du référent peuvent être conservées ou non, selon un compromis entre le réalisme ou iconicité de la reproduction et les usages assignés au modèle. L'échelle peut être modifiée, pour montrer ce qui ne se verrait qu'à la loupe ou au microscope ; une coupe peut être effectuée pour exposer l'intérieur de la fleur, du fruit, de la graine. L'artiste peut ignorer des impossibilités physiologiques et représenter sur une même branche ou côte à côte des fleurs à différents stades de floraison et des fruits à différents stades de maturité.

Pierre Lacour a souligné la divergence qui se crée au cours du XVIII ${ }^{\mathrm{e}}$ siècle entre peinture de fleurs et illustration botanique, aboutissant dans les premières décennies du XIX $\mathrm{X}^{\mathrm{e}}$ siècle à la fixation d'un idéal de la gravure botanique ${ }^{38}$. Elle se caractérise notamment par une représentation de l'allure générale du sujet, accompagnée des détails anatomiques des fleurs et des fruits. Cette illustration n'est pas le portrait d'un individu de plante, mais d'une espèce donnée : même si l'artiste montre parfois des caractéristiques individuelles du sujet représenté (le cas souvent relevé est la présence de feuilles ou fleurs flétries), les variations qui pourraient compromettre l'identification sont soigneusement évitées, car ce qui est donné à regarder, le signifié, est le taxon, l'espèce.

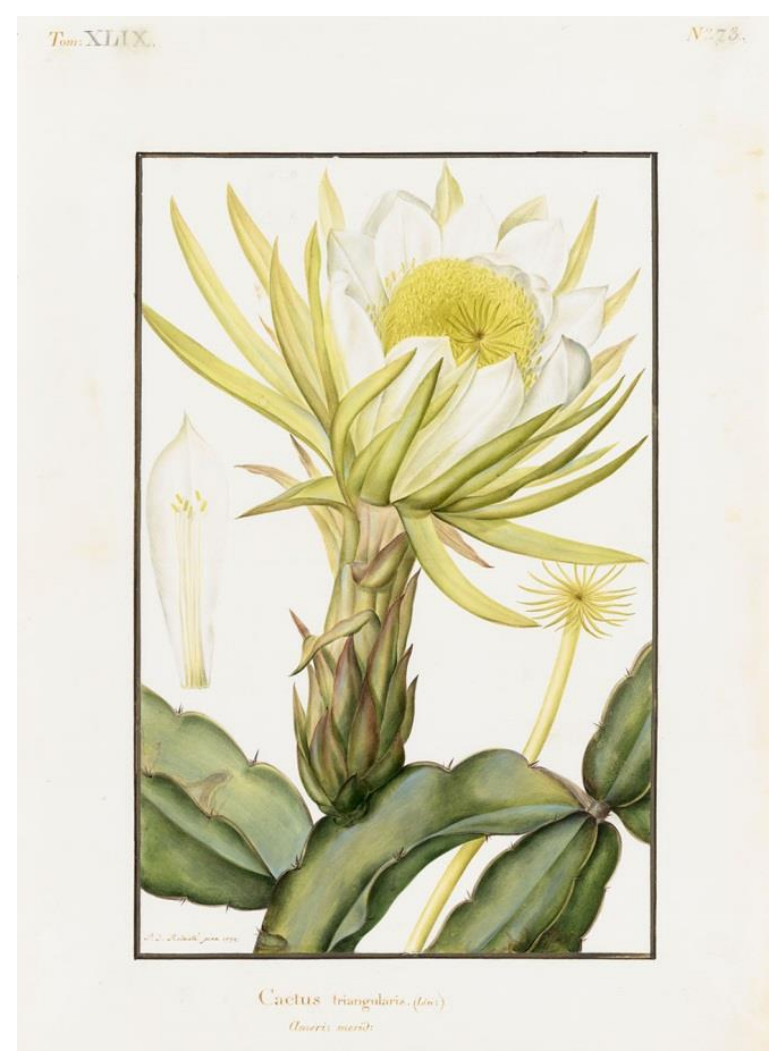

Pierre-Joseph Redouté, Hylocereus trigonus (Haworth) Safford (Cactacées) : Cactus triangularis Linné, Amérique méridionale, 1794, Collection des vélins, portefeuille 49, folio 73 ; crédit : Muséum national d'histoire naturelle, Dist. RMN/Tony Querrec

\footnotetext{
${ }^{36}$ Suzanne Bachelard, Quelques aspects historiques des notions de modèle et de justification des modèles, s.I., Maloine, 1979, 19 p.

${ }^{37}$ Henri Reiling, « Beter dan de natuur » dans Jan Brand et Alex de Vries (eds.), NEO, Utrecht., Centraal Museum, $2003, \mathrm{p}$. 221- 235.

${ }^{38}$ P.-Y. Lacour, « De la divergence art/science. L'image naturaliste entre 1720 et 1820 », art cit.
} 
Ce cahier des charges de l'illustration botanique est également suivi dans les modèles botaniques de la même période. Il correspond à la botanique telle qu'elle est enseignée alors : une science descriptive et systématique dont le principal enjeu est l'identification et la classification des espèces connues et inconnues, grâce à l'observation des caractères extérieurs, particulièrement des organes sexuels. L'exactitude de la représentation est donc essentielle : les modèles sont reproduits à leur taille naturelle, et pourraient passer pour des plantes vivantes, une impression accentuée lorsqu'ils sont présentés en pots comme les modèles de plantes du Museo di storia naturale de Florence ou les plantes artificielles de Wenzel ou de Mme de Genlis. Ils comportent une entorse à l'iconicité, nécessaire pour représenter les caractères de détermination : des fleurs disséquées pour en montrer les pistils et étamines sont représentées à côté des modèles florentins et des fleurs de verre d'Harvard ; les fruits et noyaux sont montrés en coupe dans le Carporama de Robillard d'Argentelle.

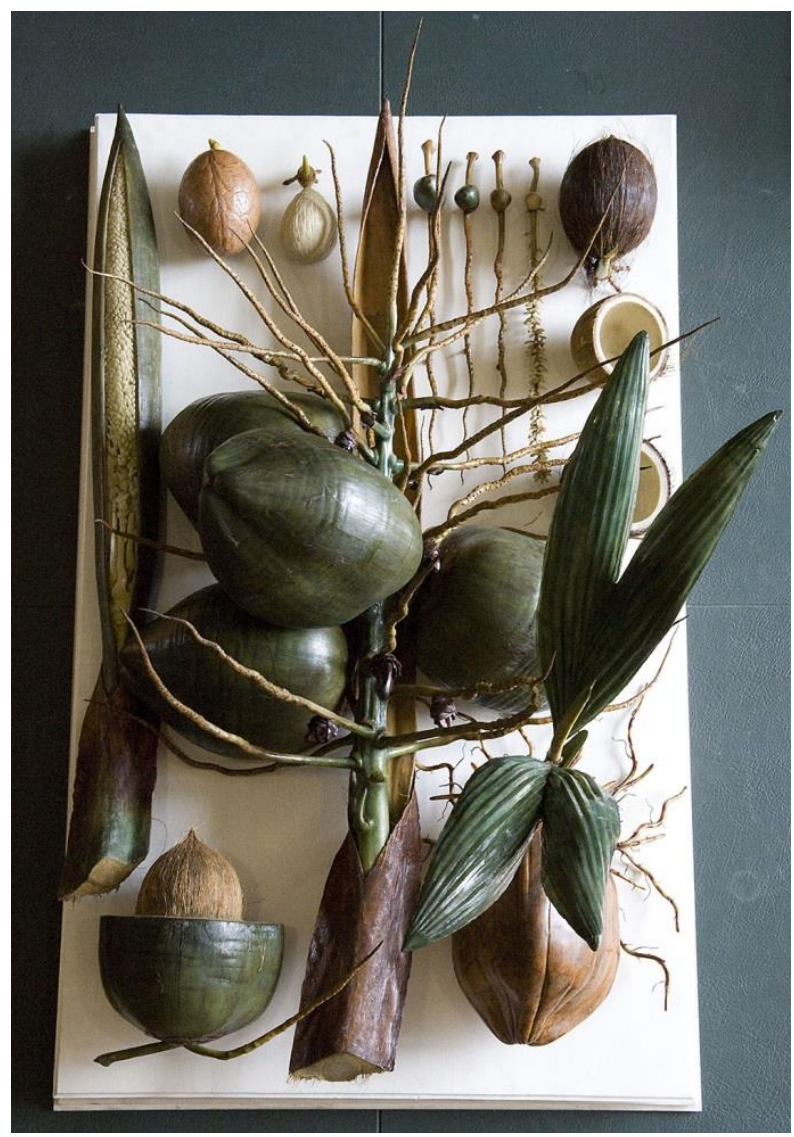

Louis-Marc-Antoine Robillard d'Argentelle, Cocotier de mer, entre 1802 et 1826, cire, bois, métal, Muséum national d'histoire naturelle, OA 1300 (002) ; crédit : Muséum national d'histoire naturelle.

Ce compromis entre un art d'imitation de la nature, cherchant à la fois à remplacer la plante vivante et à en représenter une beauté et une vérité idéales, et une science qui cherche déjà à voir à l'intérieur de la fleur ou du fruit, va évoluer vers un plus grand poids de l'exigence scientifique.

A partir du milieu du $\mathrm{XIX}^{\mathrm{e}}$ siècle apparaissent des modèles qui s'éloignent de cette représentation iconique : les modèles Auzoux, à échelle très agrandie (10:1 à $40: 1)$, ne peuvent être confondus avec de vraies plantes : la représentation a été simplifiée au profit des seuls détails pertinents pour l'observation mais surtout la compréhension de l'anatomie de la plante.

Ces modèles répondent à une évolution de la conception de la science botanique française, également visible dans les manuels de botanique à partir des années 1820 : la base à acquérir n'est plus seulement la classification ou la reconnaissance des genres et espèces, mais comprend aussi la 
connaissance de l'anatomie, nommée « organographie végétale » ${ }^{39}$, et de la physiologie des plantes. Ce changement initié en France dès l'intégration de la «physique végétale » par Desfontaines ${ }^{40}$ dans les cours de botanique du nouveau Muséum national d'Histoire naturelle se traduit en 1857 seulement dans l'intitulé de la chaire de « Botanique et physiologie végétale » du Muséum, devenant «Botanique, organographie et physiologie végétale » en 1874.

Les modèles Auzoux de la fleur de chrysanthème, du gland germé ou de la cosse de pois n'ont pas pour signifié les genres Chysanthemum, Quercus ou Pisum mais l'anatomie florale de la famille des composées, le processus de germination des dicotylédones ou celui de la croissance de la graine des légumineuses dans sa cosse. Présentant le végétal en coupe et avec un fort grossissement, ils modélisent aussi le processus de dissection et l'utilisation du microscope, devenu au XIX ${ }^{\mathrm{e}}$ siècle un outil essentiel du botaniste.

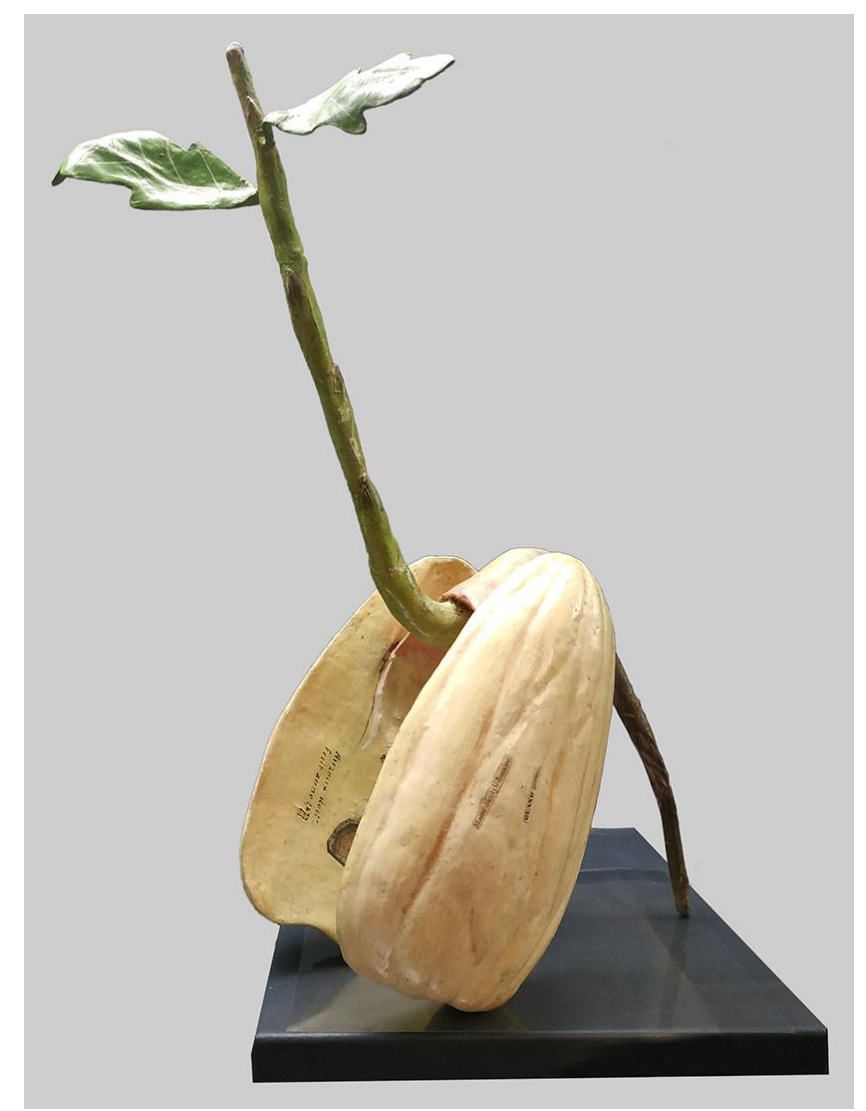

Louis Auzoux, Gland germé, 1877, papier mâché, Musée national de l'éducation, Rouen ; crédit : F. Tessier.

Les modèles Auzoux ou Brendel sont également conçus pour être démontables («clastiques » selon le terme choisi par le Dr Auzoux), non seulement par l'enseignant mais par les élèves euxmêmes. Leur appréhension par l'utilisateur est donc tactile et intellectuelle autant que visuelle, ce qui les destine particulièrement à l'usage didactique qu'ils ont parfois conservée jusqu'à nos jours. Mme de Genlis et les auteurs de manuels victoriens cités par Ann B. Shteir font aussi appel à une pédagogie active en proposant l'activité manuelle de confection des fleurs artificielles comme prétexte à observer et mémoriser l'apparence générale et les détails anatomiques de différentes espèces de plantes.

\footnotetext{
39 Le terme apparaît en 1820 dans l'Iconographie végétale de Pierre Jean François Turpin (1775-1840) formant la $3^{\mathrm{e}}$ partie des Leçons de Flore de Jean Louis Marie Poiret (1755-1834).

${ }^{40}$ René Louiche Desfontaines (1750-1833), professeur de botanique au Muséum de 1786 à 1833.
} 
Les herbiers artificiels de Marie Fortier ne relèvent pas de cette approche pédagogique : destinées uniquement à être observés, et devant servir à reconnaître des plantes communes ou utiles, ils n'offrent pas non plus de détails anatomiques servant à comprendre la vie de la plante. Cette pauvreté en discours scientifique, ou plutôt ce décalage avec ce qu'est devenue la botanique, pourrait être une des raisons du peu de retentissement qu'ils ont eu dans la communauté scientifique elle-même, et leur perte de rôle de modèle scientifique. A la fin de sa carrière, Marie Fortier commercialise ses fleurs artificielles comme modèles pour des cours de dessin, illustrant ainsi l'ambivalence du mot modèle qui peut à la fois désigner la copie du réel et ce qui est destiné à être copié.

La production de type industriel et la diffusion en grande quantité des modèles Auzoux ou Brendel, à laquelle aspire également Marie Fortier, accompagnent aussi une évolution de la place de la botanique dans la société. Réservée à une élite sociale et intellectuelle au XVIII ${ }^{\mathrm{e}}$ siècle, elle se démocratise largement au XIX ${ }^{\mathrm{e}}$, et intègre les programmes scolaires de l'enseignement primaire des lois Ferry.

\section{Muséalisation des modèles botaniques}

La poursuite de l'évolution des sciences naturelles vers une biologie portant sur l'ensemble du vivant a souvent rendu obsolètes les modèles botaniques au cours du $\mathrm{XX}^{\mathrm{e}}$ siècle. Ils ont quitté les salles de cours pour les vitrines de musées de différentes façons.

Les modèles qui dès leur conception, de par leur unicité, leur recherche esthétique et leur plus haut degré d'iconicité, étaient déjà appréciés comme œuvres d'art en plus de leur fonction didactique ont été exposés dès l'origine dans des musées d'histoire naturelle, où ils sont parfois restés au sein des mêmes institutions, comme les cires de champignons du Muséum, les modèles de verre de Harvard ou ceux de cire de Florence : seul le regard porté sur eux et leur rapport au discours scientifique a évolué.

Des plantes artificielles en cire ou en tissu ont aussi été produites pour des dioramas d'histoire naturelle, un dispositif muséal qui a connu son apogée dans la première moitié du $20^{\mathrm{e}}$ siècle $^{41}$. Après une période de discrédit, les dioramas semblent attirer de nouveau l'attention, soit comme inspiration pour l'art contemporain, comme en témoigne l'exposition de 2017 au Palais de Tokyo à Paris ; soit comme objet patrimoniaux en eux-mêmes : ainsi de l'inscription à l'Inventaire des monuments historiques des dioramas du Musée de la Porte Dorée à Paris.

Dans un diorama d'histoire naturelle représentant un biotope, les plantes artificielles devraient jouer un rôle essentiel, puisque, outre leur rôle d'acteur de ce biotope, elles permettent de compléter le fonds peint et de donner une troisième dimension au milieu naturel présenté. Le coût qu'elles représentent est parfois esquivé en choisissant de montrer des biotopes qui comportent peu de végétation: (banquise, désert, prairie sèche, haute-montagne) ou des saisons (automne, hiver) qui permettent de les remplacer par des plantes sèches. Le focus est souvent mis sur l'animal, les plantes étant perçues comme un décor plus qu'un sujet, selon le biais connu de la « cécité botanique ${ }^{42}$ ou invisibilité des plantes.

Néanmoins, la production de plantes pour dioramas a permis la survivance de quelques artistes spécialisés en modèles de plantes jusqu'à nos jours, surtout dans le monde anglo-saxon ; mais même ces productions perdent leur rôle de modèle scientifique dès lors que l'objet "plante artificielle » n'est plus utilisé, identifié ou regardé, comme la représentation d'une espèce donnée.

\footnotetext{
${ }^{41}$ Collectif et al., Dioramas, Paris, Flammarion, 2017, 347 p.

${ }^{42}$ https://fr.wikipedia.org/wiki/C\%C3\%A9cit\%C3\%A9 botanique
} 
Pour les modèles produits en série de façon industrielle comme les modèles Auzoux et Brendel, l'accès au statut patrimonial a été plus tardif et encore timide. S'ils ont été plus facilement sauvegardés dans des musées d'histoire naturelle, ils ont connu des sorts plus aléatoires dans les établissements d'enseignement primaire, secondaire ou universitaire, pourtant plus susceptibles d'en avoir acquis: chaque déménagement ou chantier de rénovation a pu être l'occasion de se débarrasser de matériel pédagogique périmé.

Leur production de masse sur une longue période avait pourtant permis leur large diffusion dans le monde entier. L'attention récente portée au matériel pédagogique ancien permet malgré tout d'en retrouver de nombreux exemplaires dans divers types d'institutions, et des projets de recensement sont en cours. Les journées d'études sur «La botanique et ses objets d'enseignement » à l'occasion de l'exposition «Belles plantes» du Munae ${ }^{43}$ ont permis de rassembler des institutions qui cherchent aujourd'hui à les valoriser. Dans certains cas, les modèles sont encore manipulés et utilisés dans le cadre de cours de physiologie végétale par les universités qui en conservent, comme à l'Université de Lille, l'Université de Bourgogne ou l'Université de Strasbourg. Mais ils sont aussi de plus en plus exposés non seulement comme pièces de musée, mais aussi comme inspiration pour la création contemporaine, comme en témoignent l'année et le site consacrés par la Haute Ecole des Arts du Rhin aux modèles didactiques ${ }^{44}$, ou l'exposition «Jardins » au Grand Palais en 2017 où figuraient plusieurs sortes de modèles botaniques ${ }^{45}$.

Quant aux modèles de Marie Fortier achetés pour les écoles parisiennes, ils semblent bien avoir tous disparus, victimes à la fois de leur faiblesse en tant que modèle scientifique, de leur fragilité, et peut-être de leur trop proche parenté avec les fleurs artificielles, accessoire féminin, frivole et fugitif. Quelques-uns sont-ils retournés à un statut d'objet sans autre but qu'esthétique, et ont-ils fini par orner des chapeaux?

Un exemplaire a pourtant survécu dans un établissement d'enseignement secondaire madrilène qui a conservé son matériel pédagogique scientifique de la fin du XIXe siècle : aux côtés de modèles Auzoux ou d'échantillons d'essences de bois, onze boites vitrées abritent des plantes artificielles montées comme des spécimens d'herbier, pourvues d'étiquettes siglées Marie Fortier et Hachette. Leur intérêt historique a été reconnu par une campagne de recensement du matériel pédagogique ancien dans les établissements d'enseignement secondaire de Madrid; le site CEIMES ${ }^{46}$ issu de ce programme a permis leur visibilité.

\footnotetext{
${ }^{43}$ Belles plantes ! Modèles en papier mâché du Dr Auzoux - MUNAÉ, https://www.reseau-canope.fr/musee/fr/connaitre/lesexpositions/exposition/belles-plantes-modeles-en-papier-mache-du-dr-auzoux.html, (consulté le 6 juillet 2018).

${ }^{44}$ http://www.hear.fr/sites/modeles-didactiques/sommaire/

${ }^{45}$ Marc Jeanson et al., Jardins, Paris, RMN, 2017, 349 p.

${ }^{46}$ http://ceimes.cchs.csic.es/
} 


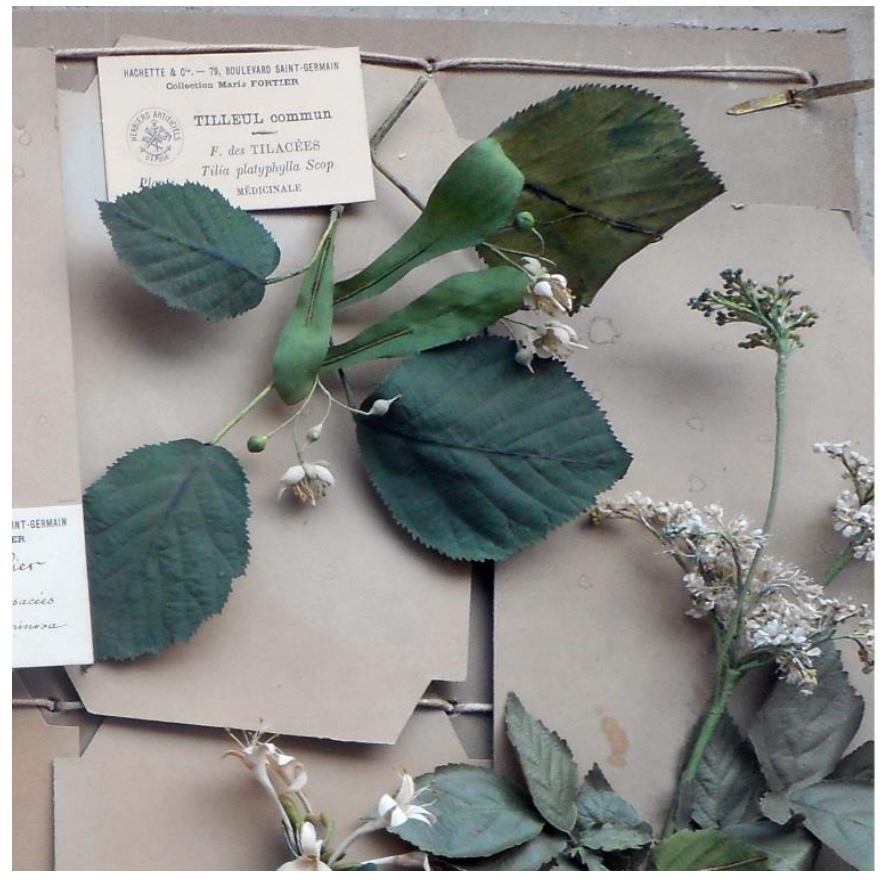

Marie Fortier pour Hachette, Tilleul commun, détail des Herbiers artificiels. 1887-1889, textile, matériaux divers. Instituto Cardenal Cisneros, Madrid ; crédit : F. Tessier.

\section{Vers une nouvelle convergence des regards?}

Entre la Renaissance et le début du XVIII ${ }^{\mathrm{e}}$ siècle, l'art et la science ont convergé et trouvé un regard commun sur les plantes et le monde végétal, avant de diverger de nouveau vers une spécialisation de l'illustration botanique. Objets d'art et objets scientifiques, les modèles de plantes en trois dimensions ont évolué de façon similaire dans cette interface privilégiée entre art et science.

Si l'illustration botanique fait l'objet d'un intérêt renouvelé dans le contexte des études sur la sémiologie des images, les modèles botaniques sont encore peu connus : le recensement de ceux qui subsistent est encore en cours, et certains types, comme les fleurs artificielles en tissu, n'avaient pas été remarqués jusqu'ici. Au-delà de leur typologie matérielle, leur diversité traduit l'évolution du discours scientifique qui influe à la fois sur la façon dont les modèles sont conçus et sur celle dont ils sont utilisés.

En cette fin du XVIII ${ }^{\mathrm{e}}$ siècle où la spécialisation du regard scientifique et la revendication par les Beaux-arts d'une esthétique de «l'art pour l'art» font diverger la peinture de fleurs et l'illustration scientifique, les arts décoratifs maintiennent le lien avec la botanique. Dans la peinture sur porcelaine ${ }^{47}$ ou le travail de la cire, du verre, de la fleur artificielle, persistent un intérêt pour une reproduction réaliste des plantes et des échanges avec la science qui les observe, les décrit, et cherche à les comprendre. Ces liens forment le contexte de modèles botaniques iconiques, produits par des artisans d'art, qui trouveront rapidement une place dans les musées ; tandis que l'adoption des procédés et matériaux industriels et la démocratisation de la science favorisent la production de modèles plus didactiques dont la muséalisation se fera plus lentement.

Le mouvement de l'Art nouveau qui s'épanouit surtout dans les arts décoratifs remet en lumière la source d'inspiration artistique que constitue la nature telle qu'elle est observée et décrite par la

\footnotetext{
${ }^{47}$ Comme le suggère l'exposition « Un jardin de papier et de porcelaine » programmée prochainement au Musée de la Manufacture de Sèvres - Cité de la Céramique.
} 
science, comme les illustrations du biologiste Ernst Haeckel ${ }^{48}$. S'emparant du regard scientifique, il s'appuie sur une connaissance savante du monde naturel, notamment végétal ${ }^{49}$.

Le regard porté sur la nature par l'écologie moderne a été similairement saisi selon Loïc Fel ${ }^{50}$ par les artistes contemporains pour créer une esthétique verte qui transparait largement dans la société actuelle. Les modèles de l'écologie, qui opèrent selon lui une présentation plutôt qu'une représentation de la nature, sont maintenant algorithmiques.

Comme Gianna Maria Gatti en donne un aperçu dans son Technological Herbarium ${ }^{51}$, de nombreux artistes actuels se sont déjà emparés de la modélisation scientifique et de la botanique algorithmique $^{52}$ pour nouer un nouveau lien entre la botanique et l'art.

\section{Bibliographie}

BACHELARD Suzanne, Quelques aspects historiques des notions de modèle et de justification des modèles, s.1., Maloine, 1979, $19 \mathrm{p}$.

BALfe Thomas, Woodall Joanna et ZiTTEL Klaus, Ad vivum?: Visual Materials and the Vocabulary of LifeLikeness in Europe before 1800, s.1., Brill, 2019.

BOGAERT-DAMIN Anne-Marie, Voyage au cour des fleurs: modèles botaniques et flores d'Europe au XIXème siècle, Namur, Belgique, Presses universitaires de Namur, 2007, 239 p.

CELNART Elisabeth, Manuel du fleuriste artificiel ou l'art d'imiter d'après nature toute espèce de fleurs, Paris, Librairie encyclopédique de Roret, 1829.

Chamagne-Rollier Brigitte, «La collection de moulages de champignons de Jean-Baptiste Barla », La Lettre de l'OCIM. Musées, Patrimoine et Culture scientifiques et techniques, 25 juin 2014, nº 153.

Chanal Nicolas, L'anatomie clastique du Docteur Auzoux, une entreprise au XIXème siècle, Thèse d'exercice, École nationale vétérinaire d'Alfort, France, 2014, 140 p.

COllectif, DoHm Katharina, GARnIER Claire, Bon Laurent Le et OsTEnde Florence, Dioramas, Paris, Flammarion, 2017, 347 p.

CORBIN Alain, COURTINE Jean-Jacques et VigARELlo Georges, Histoire du corps: De la Renaissance aux Lumières, s.l., Seuil, 2005, 668 p.

DIDEROT Denis, «Fleuriste artificiel » dans Encyclopédie, ou dictionnaire raisonné des sciences, des arts et des métiers ..., Paris, France, Briasson, 1751, vol.6, p. 866.

EXPOSITION NATIONALE. 1849. PARIS, «Fleurs de botanique artificielles » dans Rapport du jury central sur les produits de l'agriculture et de l'industrie exposés en 1849, Paris, Imprimerie nationale, 1850, vol.3, p. 647.

FEL Loïc, «L'esthétique verte: de la représentation à la présentation de la nature ».

FIORINI Graziana, MAEKAWA Luana et STIBERC Peter, «La "Collezione Brendel” di Modelli di Fiori ed Altri Organi Vegetali del Dipartimento di Biologia Vegetale dell’Università degli Studi di Firenze », Museologia scientifica, 1 janvier 2005, vol. 22, p. 249-273.

FORTIER Marie, Botanique, Notice sur les collections artificielles, s.l., Impr. de Chaix, 1885.

\footnotetext{
${ }^{48}$ Ernst Haeckel, Kunstformen der Natur, Leipzig und Wien, Allemagne, Verlag des Bibliographischen Instituts, 1899, 100; 51 p.

${ }^{49}$ Emile Gallé et al., L'amour de la fleur : Les écrits horticoles et botaniques du maître de l'Art nouveau, Nancy, Place Stanislas Editions, 2007, 324 p.

${ }^{50}$ Loïc Fel, « L'esthétique verte: de la représentation à la présentation de la nature ».

${ }^{51}$ Gianna Maria Gatti, The Technological Herbarium, Berlin, Avinus-Verlag, 2010, 246 p.

${ }^{52}$ Przemyslaw Prusinkiewicz et al., The Algorithmic Beauty of Plants, First Edition edition., New York, Springer, 1996,244 p. 
FRIGG Roman et HARTMANN Stephan, «Models in Science.pdf » dans The Stanford Encyclopedia of Philosophy, s.l., 2006.

Galle Emile, TACON François Le, VAlCK Pierre et Boulange Michel, L'amour de la fleur: Les écrits horticoles et botaniques du maître de l'Art nouveau, Nancy, Place Stanislas Editions, 2007, 324 p.

GATTI Gianna Maria, The Technological Herbarium, Berlin, Avinus-Verlag, 2010, 246 p.

GENLIS Stéphanie-Félicité Du Crest, Maison rustique, pour servir à l'éducation de la jeunesse, ou, Retour en France d'une famille émigrée: ouvrage où l'on trouve toutes les instructions nécessaires pour bâtir une maison de campagne ..., Paris, France, Chez Maradan, 1810.

GENLIS Stéphanie-Félicité Du Crest, Discours moraux sur divers sujets, et particulièrement sur l'éducation; par Madame de Genlis. Troisième édition, revue, corrigée, et augmentée d'un nouveau discours intitulé: projet d'une école rurale, France, 1802.

Guibourt, ChatTin et CAP, «Rapport fait le 2 décembre 1846 sur des plantes artificielles présentées par Mme Vény », Journal de pharmacie et de chimie, 1847, vol. 11, p. 39.

HAECKEL Ernst, Kunstformen der Natur, Leipzig und Wien, Allemagne, Verlag des Bibliographischen Instituts, 1899, $100 ; 51 \mathrm{p}$.

JAUFFRET Louis-François, Projet d'établir en France une manufacture de végétaux artificiels...rédigé par LouisFrançois Jauffret,...d'après les nouveaux procédés de T.-J. Wenzel, fleuriste de la reine, Paris, France, 1790, 120 p.

JeAnson Marc, Bon Laurent Le, Zellal Coline et HubaC Sylvie, Jardins, Paris, RMN, 2017, 349 p.

KERAUDREN-AYMONIN Monique et AYMONIN Gérard G., «Une œuvre scientifique et artistique unique: le Carporama de L. M. A. de Robillard d'Argentelle », Bulletin de la Société Botanique de France. Lettres Botaniques, 1 janvier 1984, vol. 131, nº 4-5, p. 243-246.

LACOUR Pierre-Yves, «De la divergence art/science. L'image naturaliste entre 1720 et 1820 », Études sur le XVIIIe siècle, 2015, vol. 42, p. 193-207.

LEMIRE Michel, SLEZEC Anne-Marie et BOULINIER Georges, «Un anatomiste sculpteur sur cire à l'époque révolutionnaire: André Pierre Pinson (1746-1828)»dans Scientifiques et sociétés pendant la Révolution et l'Empire (114e Congrès National des Sociétés Savantes, Section Histoire des Sciences et des Techniques, Paris, 1989), Comité des travaux historiques et Scientifiques., Paris, 1990, p. 325-337.

LEVAILlant François, Second voyage dans l'intérieur de l'Afrique par le cap de Bonne-Espérance, dans les années 1783, 84 et 85. Tome 2, Paris, H. J. Jansen, 1794.

LuDWIG David, «Mediating Objects: Scientific and Public Functions of Models in Nineteenth-Century Biology », History and Philosophy of the Life Sciences, 2013, vol. 35, n 2, p. 139-166.

Prusinkiewicz Przemyslaw, Hanan J. S., Fracchia F. D., Fowler D. R., Boer M. J. M. de, Mercer L. et LINDENMAYER Aristid, The Algorithmic Beauty of Plants, First Edition edition., New York, Springer, 1996, 244 p.

RAFFAELli Mauro (ed.), Il Museo di storia naturale dell'Università degli studi di Firenze. II, Le collezioni botaniche $=$ The Museum of natural history of the University of Florence. II, The botanical collections, Firenze, Italie, Firenze University Press, 2009, 332 p.

REILING Henri, «Beter dan de natuur» dans Jan Brand et Alex de Vries (eds.), NEO, Utrecht., Centraal Museum, 2003, p. 221-235.

SchUltes Richard Evans, DAVIS William A. et Burger Hillel S., The glass flowers at Harvard, Cambridge, EtatsUnis d'Amérique, Botanical Museum of Harvard University, 1992, vii+118 p.

SHTEIR Ann B., " "Fac-similes of nature: Victorian Wax flower modelling », Victorian Literature and Culture, septembre 2007, vol. 35, $\mathrm{n}^{\mathrm{O}}$ 2, p. 649-661.

SOCIETE BOtANIQUe DE FRANCE, «Séance du 10 février 1888 », Bulletin de la Société botanique de France, 35 (1888), nº 2, p. 103-105.

TESSIER Florence, «Les herbiers artificiels de Marie Fortier», s.l. A paraître dans les Actes du $143^{\text {e }}$ congrès du CTHS, 23-26 avril 2018, Paris.

TRIBE Henry T., « The Dillon-Weston glass models of microfungi », The Mycologist, 1998, vol. 31, p. 169-173.

TUCKWELL William, «Science-Teaching in Schools », Nature, 1869, vol. 1, p. 18-20. 
VARENNE Franck, «Histoire de la modélisation: quelques jalons» dans Modélisation, Succès et Limites : actes du colloque organisé par le CNRS et l'Académie des technologies, 6 décembre 2016, Pdf diffusé en ligne., s.1., 2018, p. 9-35.

VARENNE Franck, «Epistémologie des modèles et des simulations » dans Les modèles, possibilités et limites, Paris, Editions Matériologiques, 2014, p. 13-46.

VILLETTE Charles de, «Projet d'établir en France une manufacture de végétaux artificiels, [...]; d'après les nouveaux procédés de T.-J. Wenzel, fleuriste de la Reine, rédigé par L. F. Jauffret, In-8vo de 136 p. A Paris, 1790 ... », L'esprit des journaux, francais et etrangers, avr. 1791 p. 152-159.

Belles plantes! Modèles en papier mâché du Dr Auzoux - MUNAÉ, https://www.reseaucanope.fr/musee/fr/connaitre/les-expositions/exposition/belles-plantes-modeles-en-papier-mache-du-drauzoux.html, consulté le 6 juillet 2018. 